\title{
Anatomical and functional assessment of coronary artery disease in patients with stable angina: Which is the gold standard?
}

\author{
Federico Caobelli, MD, FEBNM ${ }^{\mathrm{a}}$ \\ a Clinic of Radiology and Nuclear Medicine, University Hospital Basel, University of Basel, Basel, \\ Switzerland
}

Received Jan 10, 2019; accepted Jan 10, 2019

doi: $10.1007 / \mathrm{s} 12350-019-01605-2$

\section{See related article, pp. 2351-2359}

Stable coronary artery disease (sCAD) is a clinical condition encompassing diverse evolutionary phases of CAD, excluding acute coronary syndromes ${ }^{1}$ and its incidence varies in parallel with observed international differences in CAD mortality, ${ }^{2}$ being generally more prevalent in middle-aged women than in men, probably due to higher incidence of microvascular disease in female patients. ${ }^{3}$

As mortality rate sank in the latest years thanks to recent advantages in diagnostic procedures and therapies, ${ }^{4}$ a prompt and reliable diagnosis is highly desirable. Additionally, the ideal diagnostic procedure should also be preferably noninvasive and provide prognostic information. In fact, the prognostic assessment is a crucial part of the management of patients with sCAD, able to identify patients who may have an improvement in outcome with more aggressive intervention, including revascularization. ${ }^{1}$

As a matter of fact, various factors can act alone or in combination to cause a clinical evident sCAD, including plaque-related narrowing of epicardial arteries, focal or diffuse spasm of coronary arteries, microvascular dysfunction, and ischaemic cardiomy-

Reprint requests: Federico Caobelli, MD, FEBNM, Clinic of Radiology and Nuclear Medicine, University Hospital Basel, University of Basel, Petersgraben 4, 4031 Basel, Switzerland; federico.caobelli@usb.ch

J Nucl Cardiol 2020;;27:2360-4.

1071-3581/ $\$ 34.00$

Copyright (c) 2019 American Society of Nuclear Cardiology. opathy with impaired left ventricular (LV) dysfunction. ${ }^{1}$ As such, a comprehensive anatomical and functional assessment is expected to yield highest accuracy in the workup of patients with sCAD.

This topic has been addressed by Akil et al. ${ }^{5}$ in this issue of the Journal of Nuclear Cardiology, wherein the authors aimed at comparing two procedures able to provide a functional assessment of CAD (i.e., cardiac magnetic resonance-CMR and positron emission tomography-PET) and an invasive technique for the anatomical evaluation of epicardial vessels (i.e., coronary angiography-CA, eventually with fractional flow reserve-FFR assessment) for the evaluation of the significance of coronary stenosis prior to transcutaneous or surgical revascularization.

The authors evaluated 41 Patients with suspected or known SCAD, who sequentially underwent a myocardial perfusion PET with ${ }^{13} \mathrm{~N}$-ammonia, CMR, and invasive CA. Of note, FFR was assessed only in a fraction of vessels (17 of $141,12.1 \%$ ).

Noticeably, the authors used PET-derived absolute quantification of myocardial flow reserve (MFR) as standard of reference, thus reporting suboptimal diagnostic performance both for CMR (sensitivity 27 to $47 \%$, specificity 75 to $96 \%$ ) and for CA (39 to $76 \%$ sensitivity and 54 to $83 \%$ specificity). These values are considerably lower than those reported in the literature. A meta-analysis by Jaarsma et al. ${ }^{6}$ including a total of 166 articles (17,901 patients) demonstrated a very good diagnostic accuracy for CMR (pooled sensitivity $88 \%$, specificity $76 \%$ ), similar to that of PET (89\% and $81 \%$, respectively). The contention may relate in the largely different patient population $(n=41$ vs 17,901$)$ as well as in that in the present study only patients with sCAD were included. But the choice of a different standard of reference represents a key factor in this regard.

The use of PET-derived absolute myocardial flow quantification (MFQ) as gold standard is fascinating and 

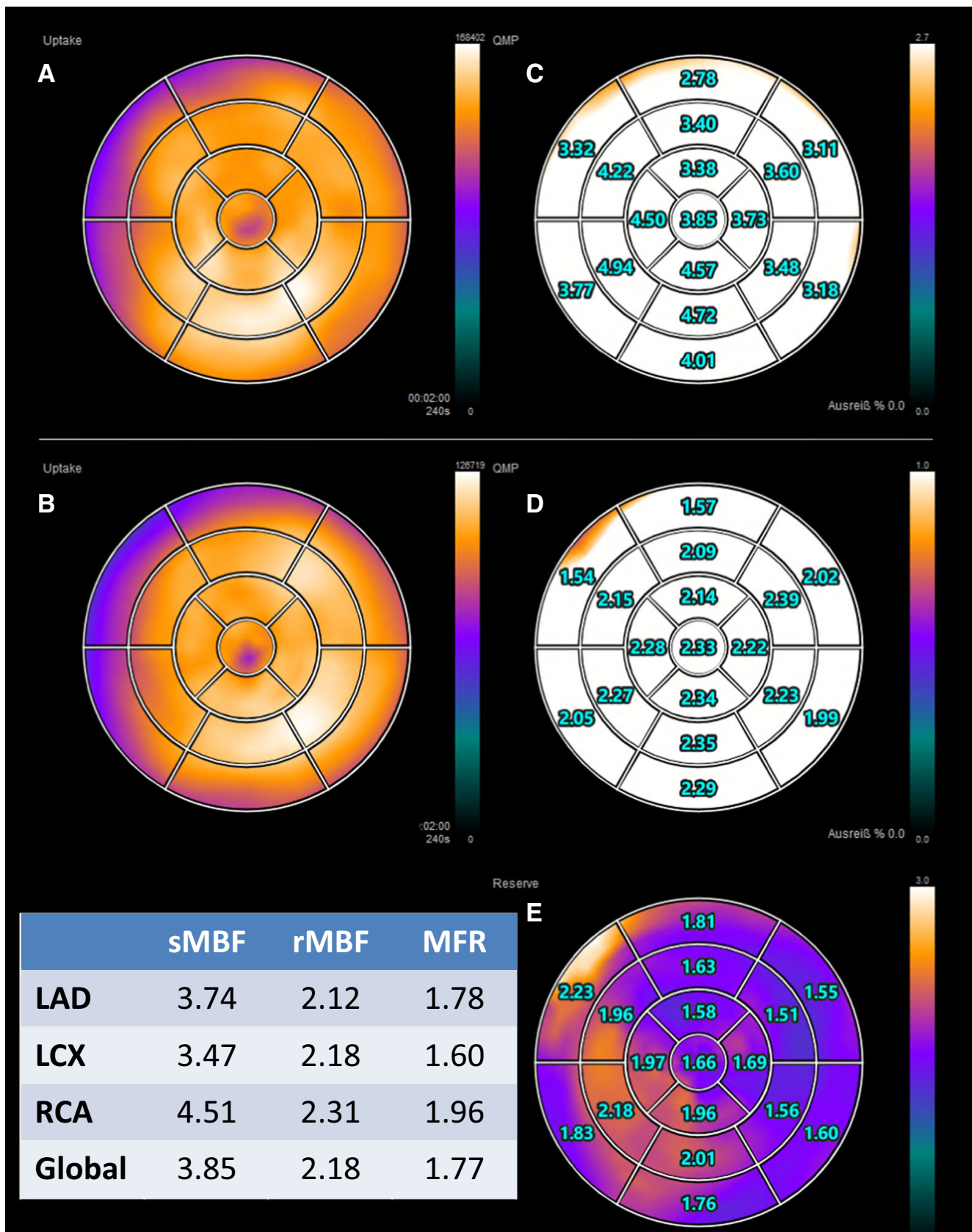

Ausreiß $\% 0.0$.0

Figure 1. Representative image showing an unremarkable myocardial perfusion PET with ${ }^{82} \mathrm{Rb}$, wherein high myocardial blood flow (MBF) at rest affects the assessment of myocardial flow reserve (MFR). Polar perfusion images at stress (A) and rest (B) show an unremarkable perfusion pattern. The MBF at stress is normal (C), while MBF at rest is above normal standards (D) due to patient's anxiety during the procedure. As a consequence, MFR values are reduced both globally and in each vascular territory (always $<2.0$, see table). Flow values are given in $\mathrm{mL} / \mathrm{g} / \mathrm{min}$. $s M F B$, myocardial blood flow at stress; $r M B F$, myocardial blood flow at rest; $M F R$, myocardial flow reserve.

supported by the fact that this information can effectively reveal a microvascular dysfunction in patients without significant stenoses of the epicardial vessels.
Unfortunately, a consensus in this regard has not been reached yet and, to date, an invasive CA with FFR assessment remains the standard of reference. 

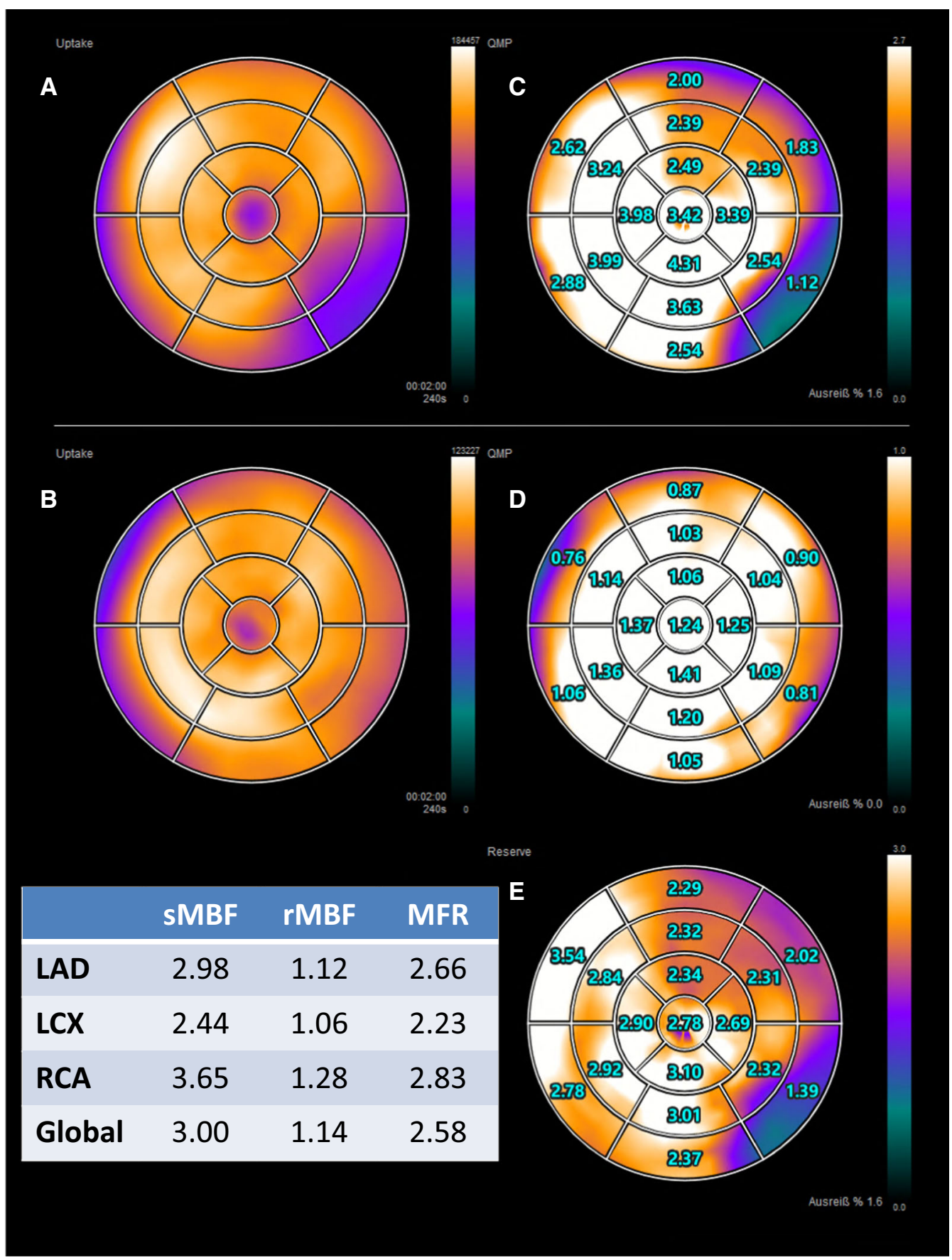

Figure 2. Representative image showing a myocardial perfusion PET with ${ }^{82} \mathrm{Rb}$, with a regional perfusion defect affecting the proximal portion of the inferolateral wall. There is a reduction of counts on stress perfusion images $(\mathbf{A})$ with recovery at rest $(\mathbf{B})$. The mean values of myocardial blood flow at stress (C) and rest (D) and the myocardial flow reserve (MFR, E) are normal both globally and in each vascular territory (see table). Specifically, MFR values are constantly over 2.0. Flow values are given in $\mathrm{mL} / \mathrm{g} / \mathrm{min}$. $s M F B$, myocardial blood flow at stress; $r M B F$, myocardial blood flow at rest; $M F R$, myocardial flow reserve.

It should also be noted that the standard of reference chosen by the authors (MFR) alone is not the most accurate parameter to predict a significant CAD.
Specifically, it was reported that in ${ }^{15} \mathrm{O}-\mathrm{H}_{2} \mathrm{O}$ myocardial PET, hyperemic myocardial blood flow (MBF) has the highest diagnostic accuracy, clearly outperforming 
MFR. ${ }^{7}$ As a matter of fact, both global and regional MFR can be affected by relatively high flow rates on rest condition (Figure 1). Furthermore, even the assessment of MFR in each vascular territory can miss localized impairments, consistent with significant CAD (Figure 2).

Other parameters like relative flow reserve (RFR), defined as the ratio of hyperemic MBF in a stenotic area to hyperemic MBF in a normal perfused area have been investigated, but an improvement in diagnostic accuracy was not demonstrated. ${ }^{8}$

Taken together, these observations suggest handling the reported diagnostic performance with caution.

The authors also fail to demonstrate a significant improvement of PET-derived MFQ in the prognostic assessment of patients with sCAD. While this goal was beyond the scope of the present paper, it should be underlined that a great advantage of myocardial perfusion imaging is the possibility to identify patients at increased cardiovascular risk, as suggested by many studies. ${ }^{9}$

Despite these limitations, the most important message from the authors is that combining anatomical and functional imaging is needed in order to yield improved diagnostic accuracy in patients with sCAD. In this regard, it should be noted that, although the anatomical assessment in the present paper relies on invasive CA, the same assessment can be effectively obtained with a coronary-computed tomography angiography (CCTA), which showed highest agreement with information coming from invasive CA. ${ }^{10}$

The potential added value of the superimposition of the coronary artery tree on myocardial perfusion in the diagnostic workup has already been demonstrated. For example, Kajander et al. ${ }^{11}$ reported that hybrid PET/ CCTA yielded superior diagnostic accuracy over standalone PET or CCTA, both on a per-patient basis and on a per-vessel basis. Furthermore, studies on combined single-photon emission tomography (SPECT) and CCTA showed important prognostic value over standalone modalities, ${ }^{12}$ thus suggesting a similar impact for hybrid PET/CCTA imaging.

It should also be noted that CCTA allows for the characterization of coronary plaques ${ }^{13}$ and its relationships with the perfusion status in the subtending territories. In this regard, the assessment of thin-cap fibroatheroma lesions by means of CCTA provides additional value over the severity of luminal narrowing. It was also shown that patients without detectable myocardial ischemia but with CCTA-defined vulnerable plaques have higher rate of future acute events. ${ }^{14}$

Future works should focus on an integrated anatomical and functional evaluation of the myocardium in patients with $\mathrm{SCAD}$, in order to assess the precise contribution of hybrid imaging to the diagnostic workup and to the prognostic assessment. Looking at the whole picture seems to be crucial for risk prediction and therapy decision.

\section{Disclosures}

The author reports no disclosures relevant to the manuscript. Specifically, no financial conflict of interest is present.

\section{References}

1. Members Task Force, Montalescot G, Sechtem U, Achenbach S, Andreotti F, Arden C, et al. ESC guidelines on the management of stable coronary artery disease. Eur Heart J 2013;2013:2949-3003.

2. Hemingway H, McCallum A, Shipley M, Manderbacka K, Martikainen P, Keskimaki I. Incidence and prognostic implications of stable angina pectoris among women and men. JAMA 2006;295:1404-11.

3. Reis SE, Holubkov R, Conrad Smith AJ, Kelsey SF, Sharaf BL, Reichek N, et al. Coronary microvascular dysfunction is highly prevalent in women with chest pain in the absence of coronary artery disease: Results from the NHLBI WISE study. Am Heart J 2001;141:735-41.

4. Benjamin EJ, Blaha MJ, Chiuve SE, Cushman M, Das SR, Deo R, et al. Heart disease and stroke statistics-2017 update: a report from the american heart association. Circulation 2017;135:e146603.

5. Akil S, Hedeer F, Carlsson M, Arheden H, Oddstig J, Hindorf C, et al. Qualitative perfusion assessment by cardiac magnetic resonance imaging and invasive coronary angiography is not enough when evaluating patients with coronary artery disease-a cardiac positron emission tomography study. J Nucl Cardiol 2019. In press.

6. Jaarsma C, Leiner T, Bekkers SC, Crijns HJ, Wildberger JE, Nagel $\mathrm{E}$, et al. Diagnostic performance of noninvasive myocardial perfusion imaging using single-photon emission computed tomography, cardiac magnetic resonance, and positron emission tomography imaging for the detection of obstructive coronary artery disease: A meta-analysis. J Am Coll Cardiol 2012;59:171928.

7. Danad I, Uusitalo V, Kero T, Saraste A, Raijmakers PG, Lammertsma AA. Quantitative assessment of myocardial perfusion in the detection of significant coronary artery disease: Cutoff values and diagnostic accuracy of quantitative [(15)O]H2O PET imaging. J Am Coll Cardiol 2014;64:1464-75.

8. Stuijfzand WJ, Uusitalo V, Kero T, Danad I, Rijnierse MT, Saraste A, et al. Relative flow reserve derived from quantitative perfusion imaging may not outperform stress myocardial blood flow for identification of hemodynamically significant coronary artery disease. Circ Cardiovasc Imaging 2015;8:e002400.

9. Monroy-Gonzalez AG, Tio RA, de Groot JC, Boersma HH, Prakken NH, De Jongste MJL, et al. Long-term prognostic value of quantitative myocardial perfusion in patients with chest pain and normal coronary arteries. J Nucl Cardiol 2018. https://doi.org/ 10.1007/s12350-018-1448-8.

10. Arbab-Zadeh A, Di Carli MF, Cerci R, George RT, Chen MY, Dewey $\mathrm{M}$, et al. Accuracy of computed tomographic angiography and single-photon emission computed tomography-acquired 
myocardial perfusion imaging for the diagnosis of coronary artery disease. Circ Cardiovasc Imaging 2015;8:e003533.

11. Kajander S, Joutsiniemi E, Saraste M, Pietila M, Ukkonen H, Saraste A, et al. Cardiac positron emission tomography/computed tomography imaging accurately detects anatomically and functionally significant coronary artery disease. Circulation 2010;122:603-13.

12. Pazhenkottil AP, Nkoulou RN, Ghadri J-R, Herzog BA, Buechel RR, Kuest SM, et al. Prognostic value of cardiac hybrid imaging integrating single-photon emission computed tomography with coronary computed tomography angiography. Eur Heart J 2011;32:1465-71.
13. Liu T, Maurovich-Horvat P, Mayrhofer T, Puchner SB, Lu MT, Ghemigian K, et al. Quantitative coronary plaque analysis predicts high-risk plaque morphology on coronary computed tomography angiography: Results from the ROMICAT II trial. Int J Cardiovasc Imaging 2018;34:311-9.

14. Otsuka K, Fukuda S, Tanaka A, Nakanishi K, Taguchi H, Yoshiyama $\mathrm{M}$, et al. Prognosis of vulnerable plaque on computed tomographic coronary angiography with normal myocardial perfusion image. Eur Heart J Cardiovasc Imaging 2014;15:332-40.

Publisher's Note Springer Nature remains neutral with regard to jurisdictional claims in published maps and institutional affiliations. 